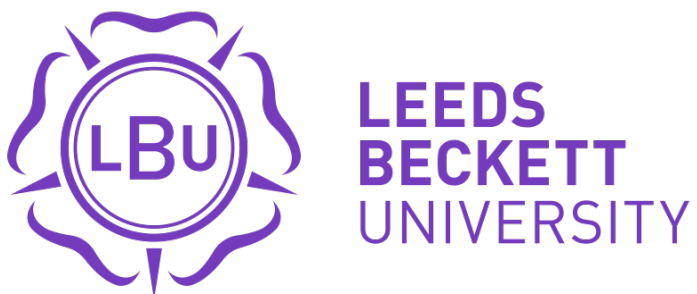

Citation:

Crowther, J and Ajayi, SO (2019) Impacts of 4D BIM on Construction Project Performance. International Journal of Construction Management. ISSN 1562-3599 DOI: https://doi.org/10.1080/15623599.2019.1580832

Link to Leeds Beckett Repository record:

https://eprints.leedsbeckett.ac.uk/id/eprint/5670/

Document Version:

Article (Accepted Version)

The aim of the Leeds Beckett Repository is to provide open access to our research, as required by funder policies and permitted by publishers and copyright law.

The Leeds Beckett repository holds a wide range of publications, each of which has been checked for copyright and the relevant embargo period has been applied by the Research Services team.

We operate on a standard take-down policy. If you are the author or publisher of an output and you would like it removed from the repository, please contact us and we will investigate on a case-by-case basis.

Each thesis in the repository has been cleared where necessary by the author for third party copyright. If you would like a thesis to be removed from the repository or believe there is an issue with copyright, please contact us on openaccess@leedsbeckett.ac.uk and we will investigate on a case-by-case basis. 


\title{
Impacts of 4D BIM on Construction Project Performance
}

\author{
James Crowther ${ }^{\mathrm{a}}$ and Saheed O. Ajayi ${ }^{\mathrm{a}, \mathrm{b}}$

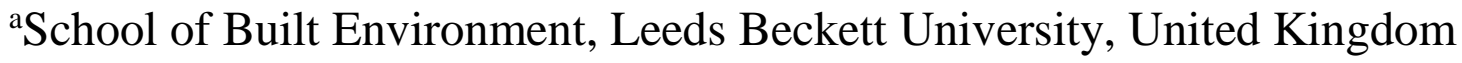

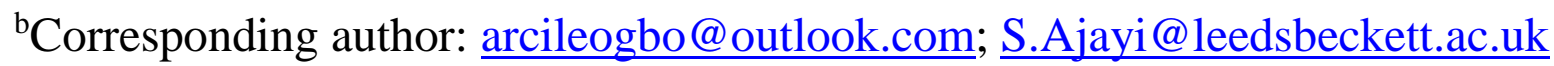

\begin{abstract}
A significant proportion of construction projects are failing to achieve their deadline finish dates. This advocate for solutions that could address the root causes of time impacting risks, leading to the use of 4D BIM for project planning. This study investigates the impacts of 4D BIM on construction projects. An exploratory sequential mixed method research was conducted to initially explore the topic via interviews and literature review, and, subsequently, the themes derived were put into questionnaires to elicit expert knowledge on a wider industry scale. The data were analysed using thematic analysis, reliability analysis, Kruskal-Wallis test and factor analysis. Across the objectives around the impacts of 4D BIM on project reliability, monitoring and diagnosis, the findings presented eight key ways the 4D BIM support project performance. Examples of component factors that were raised was planning efficiency to enhance planner output, assessment and directive with a better comparison of planned and actual progress, and thorough/comprehensive risk reflection to cover wide ranges of issues. Upon further reflection, the finding highlighted the issues of the lack of shared responsibility outside of the planner and BIM coordinator, severe lack of understanding and training regarding 4D BIM and complexity of carrying out the process effectively.
\end{abstract}

Keywords: Project Performance; 4D BIM; Project Schedule; Project Reliability; Project Diagnostics

\section{Introduction}

As an industry that is famously labelled as inefficient and inconsistent, the construction sector still suffers from issues regarding on-time delivery. The defunct efficiency had been addressed within the 1998 Rethinking Construction report claiming costs and delays incur with the design and planning stage (Egan, 1998). Even to this day, a significant proportion of projects are failing to achieve completion on their deadline handover dates, leaving clients with unusable/unfinished assets and contractors with liquidated and ascertained damages to reimburse. In the U.K alone, it was reported 
that ' $70 \%$ of government construction projects were delivered late' (National Audit Office, 2003, p. 3 ) and around a third of U.K construction projects failed to achieve their deadline finish dates.

Due to the industry issue of projects overrunning, there are several systems that have been developed to help minimise delay causing factors, one of which is known as lean construction to attain a maximum value of a project via the efforts of a fully collaborated project team. The principles of lean construction prevent project delay through thorough communication, eradication of inefficiencies/waste activities, and uses techniques such as modelling and visualisation-based planning, prefabrication and risk management procedures (HM Government, 2016). A more modern bi-product of the lean philosophy has come to fruition via the introduction of building information modelling (BIM) (Sacks et al., 2010; Zhang et al., 2018), which the industry has gradually adopted within the past years. The government has encouraged the industry's utilisation of BIM at a level 2 maturity stage which requires 'fully collaborative 3D BIM with all project and asset information, documentation and data being electronic as a minimum by 2016' (Cabinet Office, 2011, p. 14). Evidence suggests that the use of BIM in construction project facilities cost benefits (Lu et al., 2014). As the level 2 BIM is now mandatory for centrally-procured public projects, this automatically facilitates the ability to implement 4D BIM into the process, allowing planners and project managers to input programme data information into a fully federated 3D model. In essence, this allows the construction sequence to be visualised from any point in time or location, within the virtual project environment, bringing a much more immersive perspective to a project programme.

For the project teams to maintain quality performance, it is vital that the programmes produced are as reliable as possible via accurate durations, logical sequencing, identification of high-risk activities, and easy to monitor actual progress against planned progress, to better secure on-time delivery. All these elements are what 4D BIM aims to better implement, involving a much more visual and animated representation of a project programme than a solely used traditional Gantt chart. This, thus, utilises a more technological approach as recommended by the Rethinking Construction report by primarily developing the culture of the industry and assisting this with the aid of technology (Egan, 1998).

BIM has continuously grown in importance within the modern-day construction practice, with adequate content existing with regards to the fundamentals and abilities of 4D BIM. However, most of the detailed research still revolves around more primitive topics such as 3D BIM or the holistic 
BIM approach. There is currently very little literature evidence to suggest that the 4D BIM is even commonly or effectively put into practice on current day projects to implement or enhance real tangible benefits to current projects. This shows evidence of a large general knowledge gap on the matter from a practical basis, which begs the question as to whether it genuinely achieves results, or it is just a tick in the box for company technological credentials with little end value. As such, there is a need to evaluate whether 4D BIM technology is sophisticated enough to provide a strong basis for positively impacting on project performance in comparison to a more traditional planning method

In a bid to understand whether any enhancements can be implemented to projects through the utilisation of 4D BIM, this study investigates the impact of 4D BIM on project performance. According to Kerzner (2013), the planning and monitoring/control stages is very important to reinforcing success throughout the project, as it entails allocation of required resources, scheduling of activities, and comparison of actual outcome to the predicted outcome. Project reliability is an important element of project performance that considers whether the virtual simulation of the project programme can better achieve meeting planned start and finish dates in a more efficient and consistent manner. Another relevant element of project performance is to understand time benefits of the 4D BIM, which involves inspecting the effectiveness of its progress monitoring abilities. This ability is vital for assessing whether the project is on track, identifying where and why delays have occurred. The final element of project performance is the ability for 4D BIM to proactively flag up and diagnose project risks ahead of occurrence, as opposed to just being reactive with progress monitoring, and also inspect its capability for contributing solutions to these identified risks. Larson \& Gray (2011) supports the importance of project diagnosis by discussing the dangers of schedule slippage, and how early detection of risks during control phase is required to reduce the chance of small delays growing into large ones. Based on these three elements of the project performance, the study fulfils its aim of investigating the impacts of 4D BIM on project performance by evaluating its ability to enhance the following:

- Project reliability - Ability to perform and succeed as planned time wise.

- Project monitoring - Ability to keep track of all aspects of a project in a more efficient manner.

- Project diagnosis - The capacity to proactively identify issues and risks within the project and facilitate solutions for addressing it. 
As a result of the paucity of literature regarding 4D BIM application and impact on real projects, a combination of qualitative and quantitative methods was used. This involved interviewing industry professionals with 4D BIM experience to explore the topic in detail, and subsequent administration of questionnaires to wider industry professionals to test this information on a general scale for reliability. The interviews were analysed via thematic analysis to decipher key themes regarding the detailed information on 4D BIM impact. The questionnaire data were analysed via reliability analysis to ensure reliable data is processed, and factor analysis to identify the key component factors.

In order to provide theoretical insights for the study, the next section presents a review of the extant literature covering the concept of BIM and its application. The specific processes of gathering and analysing the data are then justified and discussed. Before culminating the study with the summary of the findings and implications, the findings were discussed in line with the impacts of 4D BIM on project reliability, monitoring and diagnostics.

\section{Building Information Modelling and the Construction Industry}

Before technology was greatly advocated by the U.K government to get the construction industry to adopt a more efficient working culture, the focus was initially put on creating more trusting partnerships between client and contractor teams, in order to promote a less hostile relationship and encourage a better project delivery process. Latham (1994) identified disputes as one of the major barriers to general successful project performance. The report suggests the need for collaborative and standardised procedures with the inclusion of interrelated documentation, speedy dispute resolution, commitment to best practices, and partnering. BIM has integrated this into its general protocol with all project data being centralised and easily available to all relevant project members within a Common Data Environment (CDE), helping to eliminate the scope of information loss and obscurity.

Building upon the identified problem of poor and conflicting relationships between project teams, the U.K government later addressed the issues more specifically around quality and inefficiency of the construction industry. Egan (1998) highlighted how the reluctance to modernise was still causing profitability, technology adoption, training and value tendering to suffer while also hindering project performance. The Rethinking Construction Report (Egan, 1998) came to many of the same conclusions as for the Latham Report (1994) with the call for collaborative solutions. In addition, the Egan report recognises the needs for new technology around 3D modelling and global positioning 
systems. Some of these requirements and recommendations have now been met by the advent of the BIM, which incorporates a collaborative approach as its core platform. On a similar note, planning based efficiencies were recognised as a requisite for driving efficiency in the construction industry. While the report did not claim technology to be the complete answer to tackling inefficiency, it suggested culture change was prerequisite to bringing out the true potential that technology can provide projects.

The most recent and significant U.K government-based report on the construction industry is the Government Construction Strategy 2016-20, which was revised from the previous Government Strategy 2011-2015. The overall aim set out by the 2016-20 strategy is to improve delivery efficiency and performance so as to ultimately save on costs of up to $£ 1$.7billion, and it identifies BIM as a major priority to achieving this feat (HM Government, 2016). With cost savings targeted of $£ 8$.8bn (HM Government, 2016) in the previous 2011-15 strategy, the 2016-20 strategy reported this fell massively short with efficiency savings of only $£ 3$ billion. The 2016-20 strategy bares extremely little mention of efficiencies regarding time savings despite the Construction 2025 report targeting $50 \%$ reduction in the overall time for new build and refurbished assets. With such an ambitious target, it is hard to comprehend how there is no mention of BIM potential to achieve this through the benefits of 4D BIM, which again shows that there is a lack of awareness of the benefits of using 4D BIM.

Meanwhile, BIM has been one of the most popular technical advancements the construction industry has experienced in the past decade, with around $74 \%$ of firms now utilising it according to Hardin \& McCool (2015). It has grown in significance as the knowledge surrounding its benefits on general projects has progressively been realised. The premise of BIM was due to the lack of innovation and lingering inefficiencies calling for the need for automation and technology to integrate and develop upon the traditional practices within construction. From a holistic perspective, BIM can counter these inefficiencies through the ability to carry out a project virtually before it is executed physically on site. This enables project members to carry out such responsibilities as designing, estimating, sequencing in a digital environment that bears far less consequence for errors and increased scope for amendments. BIM provides value in many ways, and it lends itself to be utilised to achieve project goals and be set up to tackle risks that may have been identified at the outset e.g. logistic planning around a site with tight constraints, simulated scheduling for organising numerous work packages or 
asset tagging for maintaining strict quality assurance. As BIM can be seen to assist all phases and roles of a project, it can be interpreted in terms of the dimensions it possesses such as 3D and 4D.

3D BIM is the foundation dimension that is associated with the graphical generation of the design from a model-based perspective. While this is not strictly a BIM feature, BIM is used to federate all the separate designed packages together within a common data environment (CDE) and contains all the property data based within each element of the model (McPartland, 2017). With BIM being centralised within a CDE, it highlights how the design process can become a much more collaborative practice as it depends on all disciplines bringing their efforts into one. This, in turn, highlights coordination issues for it to be further addressed and gain the required level of buildable quality in a timelier manner. Zhao et al. (2018) support this through their research into utilising base architectural models to devise a rapid structural model, and the evidence shows that using the parameters of the architects' $3 \mathrm{D}$ design enabled the structural design to be extremely accurate in a short amount of modelling time.

As the 3D BIM containing all the necessary property data within the graphical federated elements, 4D BIM requires this data to perform their further project management functions. 4D BIM is heavily orientated around the planning role of a project, focusing on the time, resource and logistical elements. In the 4D BIM environment, the construction programme data is linked to the 3D model, enabling a visual simulation of the construction sequence carried out on all the modelled elements. There are numerous core benefits with the 4D BIM process as proposed by Eastman et al. (2011), this includes its communication enhancement by presenting a clear and detailed plan to numerous stakeholders that would not otherwise grasp the detailed information of a Gantt chart. Site logistics are also better considered with the use of 4D BIM according to McPartland (2017), as it allows being able to integrate moving delivery loads and plant items, material laydown areas and potential obstructions. Another perceived benefit of 4D BIM is the trade coordination enhancement, highlighting the sequential flow of trades within the special constraints of the site. The final major 4D BIM benefit is through the monitoring process, being able to better compare schedules and track construction progress, using the planned virtual simulation against the actual in-situ site progress to assess the schedule status of the project. From the scarce examples of real-life project application of 4D BIM, some basic research was conducted by Ciribini et al. (2016) who carried out a case study of a residential project utilising 4D BIM to support the design and construction stages. This method of planning allowed all stakeholders to participate in the process to validate the design and avoid issues 
that would not have been identified through other means. Using 4D BIM software also allows multiple programme baselines/scenarios to be simulated so that the best solutions could be pursued.

4D BIM seems to have the most application to project performance, having fundamental benefits to the planning and monitoring/control phase of the project which are considered the key stages for addressing risk management. Upon analysis of the existing literature on 4D BIM attributes towards impacting on project performance, some authors (e.g. Larson and Gray, 2011; Eastman et al., 2011) have expressed a great amount of commendation towards it from a theoretical point of view. This is especially regarding its visual enhancement of planning, which in turn has created numerous risk management benefits and positive influence upon collaboration as per the original U.K government recommendations previously identified. Since the literature is extremely theory-based, there is a strong sense that 4D BIM bares little practical benefit to current day projects, especially as there is a very little mention of genuine case study examples. It is, therefore, important to evaluate its impacts based on the lived experience of the experts.

\section{Research Methods}

As 4D BIM and its impact on project performance had scarcely been investigated, an exploratory sequential design was deduced as the better option. With the aim being centred on a technical subject such as 4D BIM with specific objectives relating back to the application in a practical context, indepth information was required to develop a detailed understanding of its various aspects. Fellow \& Liu (2015) state that to develop construction knowledge on nascent subjects, an exploratory approach should be used. The aim also contained various elements of project performance, which could mean different things to different people. As such, to satisfy the broad nature/demographic of construction projects, general perspectives should subsequently be considered through a quantitative approach.

\subsection{Qualitative Data Collection and Analysis}

In the first phase of the exploratory sequential mixed method research, interviews were used as a means of collecting qualitative data. This is especially as the lived experience of the participants was necessary to develop insight into the research topic. Likely issues such as the participants not being equally articulate or perceptive were reduced due to the interviews being conducted with competent professionals within their typical work environment. Bias was also controlled by avoiding leading questions and interpreting the information as direct to the verbatim transcription. The interviews 
were all circa one hour long to conduct and were carried out within the participant place of work to keep a familiar setting for natural validity. Also, being at the typical work environment of the participant, it allowed them to use resources to aid their explanations with documents and slideshows for all the participants, as recommended by Creswell \& Creswell (2018). Although focus group discussions could have possibly achieved a similar outcome, it was avoided as it could cause some participants to be more reserved about their opinions within a group setting with more knowledgeable others.

Using convenient sampling as recommended by Merriam (1998), the research population was selected on the basis of including the most relevant target audience for the subject matter of 4D BIM in practice. With this being established as a highly collaborative process, this also meant numerous roles could be selected regarding their work base, profession and skill set. The most relevant roles of construction planners and BIM coordinators were chosen due to their expertise in planning and technology-based background knowledge. A population of six was sought for the qualitative research to gain in-depth accounts of respondents' lived experience of the 4D BIM. An effort was made to prevent the generalisation of a certain role experience; hence, three planners and three BIM coordinators were selected. Fellows \& Liu (2015) claim that around 6 - 10 interviewees would be required for gathering the in-depth data, which was achieved. The experience of the respondents in the AEC industry ranges from 14 to 29 years.

To evaluate the detailed information regarding the impact of 4D BIM on project performance, all the interviews conducted were transcribed to a written document. Having these interviews documented enabled the possibility to conduct a thematic analysis to identify both implicit and explicit statements that emanated from the written data (Braun \& Clarke, 2006). The highlighted themes picked up during the interviews were subsequently processed through a coding scheme to identify the most common themes expressed amongst the interviews. While the coding scheme enables an all-inclusive processing strategy of the interview data (Braun \& Clarke, 2006), this also implemented more focus on the issues that the industry really considers the most important matters regarding the impact of 4D BIM on project performance. Table 1 shows an example of how this was conducted with the some of the most prominent themes/factors that were established.

Insert Table 1: Thematic analysis results identifying the relevant areas to address for the questionnaire 
Based on the thematic analysis and coding scheme process conducted on all the interview data, a summary of the themes that were identified from this and the literature review are presented in Table 2.

Insert Table 2: Identified themes of 4D BIM regarding the impact upon project performance

\subsection{Quantitative Data Collection and Analysis}

The identified themes, shown in Table 2, were used to develop the questionnaire to further the data collection from a wider population within the construction industry. Due to the paucity of literature regarding practical examples of 4D BIM impact, numerous new themes were included to show areas of further positive or negative impact. Upon analysis of the themes extracted from the literature review, there are a considerable number of areas that the interviews were unable to validate.

The administration of these questionnaires was done through emailed web links that could be forwarded on to a larger scale of relevant participants. 50 respondents answered questions within 5 sections, including the general background of the respondent, the second, third and fourth to understand 4D BIM impact on the objectives (project reliability, monitoring and diagnosis), and fifth to express further information about 4D BIM. Table 3 provides an overview of the research respondents.

Insert Table 3: Demography of the respondents

\subsection{Quantitative Data Analysis and Findings}

The resulted quantitative data was analysed using reliability analysis, Kruskal-Wallis test and exploratory factor analysis which were carried out using SPSS version 24. They are as discussed below.

\subsubsection{Reliability Analysis}

Before the factor analysis is conducted, reliability analysis should precede this so as to clean the data by removing factors that might not be contributing towards the overall reliability of the data (Chen \& 
Hooper, 2012). As the questionnaire is based on a Likert scale, the numerical data can be processed to give the Cronbach alpha coefficient which measures the internal consistency of the data (Nunnally \& Bernstein, 1994; Yockey, 2011). Inputting these into SPSS version 24 determines whether a sufficient coefficient is achieved, with a value of 0.8 being considered a very good level of internal consistency as claimed by George \& Mallery (2003). This means that the Cronbach alpha of 0.815 achieved for the study shows a good level of internal consistency. The reliability analysis can then identify which items in the questionnaire is not contributing to the overall internal consistency through "Cronbach's alpha if item deleted" as suggested by (Field, 2013) meaning that any item with a Cronbach's alpha value above 0.815 is a poor construct and is excluded from the list and from any further analysis. 11 variables (F4, F11, F15, F17-F20, F26, F31, F42, F44) exceeded this Cronbach's Alpha, which when excluded improved the Cronbach's alpha coefficient to 0.840 .

\subsubsection{Kruskal-Wallis Test}

To determine whether there is a difference in respondents' perception of the factors based on their job roles, a Kruskal-Wallis test was carried out as recommended by Gupta (2010). While a p-value (Asymp. Sig) below 0.05 would reveal a noteworthy difference in the perception of the respondents, the findings of the study show that the respondents do not differ based on their role, as none of the factors has its Kruskal-Wallis coefficient less than 0.05. This suggests that the finding represents a general view of the respondents irrespective of their job roles and that the data from the different professions could be combined for further analysis.

\subsubsection{Exploratory Factor Analysis}

Factor analysis is a 3-step process with the suitability of data, factor extraction and factor rotation. The suitability of data required numerous procedures with Kaiser-Meyer-Olkin (KMO) requiring a sampling adequacy above 0.5, Bartlett's test of sphericity requiring a value below 0.05 and determinant of coefficient matrix tests valuing above 0.00001 (Field, 2013; Tabachnick \& Fidell, 2001). The KMO is a measure of sampling adequacy that determines whether the data is suited for factor analysis (Field, 2013). The Bartlett's test of sphericity assumes that the correlation matrix is an identity matrix; and as such, a value above 0.05 (non-significant value) means that the variables are unrelated and unsuitable for factor analysis (Tabachnick and Fidell, 2001). The determinant of coefficient matrix, on the other hand, is used for testing for multicollinearity in the data. The factor extraction procedure was conducted via Principal Component Analysis (PCA) requiring an Eigenvalue of 1 (Nunnally \& Bernstein, 1994) along with a diagonal matrix value below 0.5 (Field, 
2013) to remove any further required factors. The factor rotation procedure conducted was the orthogonal Varimax method to redistribute factors and retain the group components.

Project reliability was the first objective tested via factor analysis, using SPSS version 24 to determine a KMO value of 0.744 , Bartlett test of 4.276E-20 and coefficient matrix of 0.005 all of which satisfy the required values and are appropriate for factor analysis based on the three coefficients. The factor groups were determined and named based on the common underlying factors relating them together. With the factor groups now established, the use of the Eigenvalue and percentage of variance is able to determine the order of the key factors.

Project monitoring was the second objective to be processed via factor analysis, using SPSS version 24 to determine the KMO value at 0.688 , the Bartlett test coming to $1.1211 \mathrm{E}-21$, and coefficient matrix at 0.011 , again acquiring sufficient values across the board. Despite initially generating three component groupings and further processing with the orthogonal Varimax method of rotation, one group is only able to include the lone factor 32 '4D BIM is best used with site time-lapse cameras for identifying actual progress', meaning this group is null and excluded. A value was unable to be assigned to factor 25 'It's important that the level of detail in the 4D BIM model genuinely and accurately matches what's on-site', leading to the removal of the factor.

The last objective to process through factor analysis was the projects diagnosis. With the SPSS version 24 presenting a $\mathrm{KMO}$ value of 0.733 , Bartlett test of $7.6006 \mathrm{E}-17$ and coefficient matrix 0.021, this also shows a comprehensive satisfaction of all the data suitability for factor analysis. Only three component groupings can be established (as opposed to an initial 4) due to the orthogonal Varimax method of rotation process highlighting the lone factor 34 'Clash detection is frequently used in conjunction with 4D BIM to highlight logic/sequencing errors', meaning that the group 4 is to be excluded. A value was unable to be assigned to factor 33 ' $4 \mathrm{D}$ BIM is useful for conflict resolution and contractual disputes' leading to the removal of the factor. Table 4 presents the result of the factor analysis

Insert Table 4: Component labelling of the related 4D BIM impact factors on project 


\section{Discussion}

Findings from the exploratory factor analysis are discussed in this section.

\subsection{Impact of 4D BIM on Project Reliability}

The impacts of 4D BIM on project reliability are as discussed below.

\subsubsection{Group A1 - 4D BIM Planning Efficiency}

This component group was the highest rated total variance of the 3 categories for project reliability at $25.401 \%$ as shown in Table 4. '4D BIM Planning Efficiency' was the name chosen for this component factor based on the related factors suggesting 4D BIM is a simple and effective way to conduct planning responsibilities. For example, easy phase splitting as proclaimed by Hardin \& McCool (2015) and its generation of multiple scenarios being a simple process (Mordue \& Finch, 2015). With planning efficiency being the highest rated impact factor for project reliability, this suggests that there is a strong general approval of the process amongst project teams to get the project plan executed right upfront early in the project. In addition, more utilisation of the 4D BIM tools and features over typical critical path method (CPM) based planning may be the key factor achieving more success regarding project performance, building on the point by Hardin \& McCool (2015) which claim that the CPM planning is less efficient. Due to this being the most relevant component factor identified for project reliability, this signifies that for 4D BIM to really bring this efficiency into fruition. It is expected to include more functions to allow other roles to share more responsibility, as even though the planning functions are presented as more efficient, the planner is still the only role championing them, meaning more training is required to the likes of project/site managers/engineers to share the planning responsibility. This could encourage more collaborative process and could lighten the planner workload, which according to this component factor should not be an issue with the sufficient efficiency level.

\subsubsection{Group A2 - 4D BIM Collaboration \& Education}

The finding also shows that the collaboration and education as high impact factor on project reliability, having a total variance of $21.547 \%$ as shown in Table 4 . The component name of '4D BIM Collaboration \& Education' was based on the incorporated factors all regarding the involvement of different roles, even from external project members such as clients and demolition subcontractors (Eastman, et al., 2011). The link with education comes with two different perspectives, firstly the demand for more familiarisation as suggested by Han \& Golparvar-Fard 
(2015), which can only realistically come with training and consistent utilisation of the process on a project. The second point is the learning implications of the process, being able to analyse and explore complex project phases such as demolition works from a virtual setting, to significantly understand the planned activities and their implications. This suggests that the Hardin \& McCool (2015) statement on encouraging more collaborative utilisation cannot just only rely on the main roles of planners and BIM coordinators receiving the necessary training, but the majority of project roles. For instance, an involvement of the client representative and the quantity surveyors could help further spread the understanding of the process and allow collaboration to thrive with multistakeholder input, thereby gaining a higher value output to increase the reliability of capitalising on project benefits.

\subsubsection{Group A3 - 4D BIM Progress Continuity/Consistency}

The last relevant component factor based on 4D BIM impact on project reliability is classified as '4D BIM Progress Continuity/Consistency' at a total variance of $14.447 \%$ as shown in Table 4 . This factor grouping was named on the principle of its factors being relevant to the important contribution that the 4D BIM provides by driving projects forward and making sure progress is retained throughout all the necessary stages as claimed by Eastman et al (2011). This is more of a general reflection from the respondents that 4D BIM has developed significantly as a tool, and it has matured enough that once implemented would be hard to come away from in future stages of the project or even subsequent projects. This was reflected by Higgin \& Cousins (2013) who suggest the detrimental effects of having to resort back to the non-BIM based solution. The implications of these factors would be that to allow 4D BIM a platform to deliver this project continuity/consistency there is a need for early implementation and contractual security to enforce the utilisation throughout. This is implied in Latham (1994) argument for collaborative efforts upfront to secure the necessary agreements and documentation to back the process up. At the very least, there should be compensatory measures for being unable to employ these efficiencies, as reflected by Higgins \& Cousins (2013) with Z clauses.

\subsection{Impact of 4D BIM on Project Monitoring}

The impacts of 4D BIM on project monitoring are as discussed below.

\subsubsection{Group B1 - 4D BIM Assessment and Directive}

Out of the two-component categories identified in the factor analysis segment for project monitoring, this was the highest regarding the total variance being $31.047 \%$ as presented in Table 4 . The 
component factor was named '4D BIM Assessment and Directive' considering the factors being based around allowing the user to analyse the actual progress in direct comparison with the planned progress as claimed by Hardin \& McCool (2015). This suggests the emphasis on the quantity and quality of assessed progress data is not only able to be better accommodated using 4D BIM than more traditional means but is also a critical requirement for enabling the process to provide benefits to the project, even information regarding small - medium plant items as claimed by Eastman et al. (2011). Being able to better assess the project progress enables a much more informed decisionmaking process, with a clearer vision of how to address the further activities based on well documented recent past information. This suggests that there is a great amount of interest in being able to justify the methods of moving a project forward. This aligns with Eastman et al. (2011) point on using the data input function to better project planning, and the recording and utilisation of the existing data of previous stages could be an extreme influence for adopting 4D BIM on a project, hence the ability to do this needs to be more accessible to the user to carry out.

\subsubsection{Group B2 - 4D BIM External Technology and Logic Utilisation}

The other component factor recognised for impact on project monitoring was through being able to enhance the utilisation of other technologies, whilst still being able to apply expert logic to further enhance the monitoring process. This came with a total variance of $23.985 \%$ as shown in Table 4 . The component was named '4D BIM External Technology and Logic Utilisation' on the basis that the factors highlighted how the combination of human logic and data-rich technologies can be brought together through 4D BIM to once again make more informed decisions to take the project forward. This implies that despite the fact that 4D BIM is primarily focussed on proactively addressing risks, it also has the ability to address issues reactively as an alternative method of control. This corresponds to an earlier claim by Eastman et al (2011) that the system can help to analyse unforeseen circumstances. Such proactive and reactive activities could be carried out during the monitoring phase by the 4D BIM user to explore all the parameters of the issue with the monitored information, picked up from the likes of 3D point cloud scans input with the 4D BIM model (Han \& Golparvar-Fard, 2015). While it highlights the importance of how technology is seen as a necessary influence, enhancing the way a project can be perceived and managed, it also shows there will still be a great reliance on expert knowledge to truly interpret and act on the information gathered by the technology. This means that human input is still depended upon, which in turn requires 4D BIM to be as user-friendly as possible. This issue was also raised during the interview, leading to the assumption that while the idea of technology assistance could be useful, it just has not 
yet taken off; hence, more training with the appliances in conjunction with the 4D BIM process is required to reduce the overdependence of planning logic and margin for human error.

\subsection{Impact of 4D BIM on Project Diagnosis}

The impacts of 4D BIM on project diagnosis are as discussed below.

\subsubsection{Group C1 - 4D BIM Thorough/Comprehensive Risk Reflection}

The impacts of 4D BIM on project diagnosis is identified to be most contributed towards through the ability to delve deep into identifying and mitigating risks, where it had the highest total variance, out of the three component factors, at $22.99 \%$ as shown in Table 4 . The name for the component group is '4D BIM Thorough/Comprehensive Risk Reflection' as all the factors involved include a wide spectrum of how 4D BIM addresses risks. The factor suggests that the project risks can be identified, through the 4D BIM and it would allow the users to specify what the risk is as covered as also suggested by Mordue \& Finch (2013). This suggests that the project teams are very interested in how 4D BIM can apply itself into not only highlighting risks but also involving the necessary resources to help address the issue across all relevant stages in the project, as Hardin \& McCool (2015) point on still being useful in the upfront stages. This shows a strong indication relating back to collaboration having to be the main aspect that 4D BIM needs to accommodate for. This would allow for numerous roles to be involved in the process, and then also have the expertise to identify risks simulated by the 4D BIM model in relation to their role, that other members may not have been able to. For instance, a planner and designer have a different risk agenda with time and quality, but a quantity surveyor alongside this process will give an added mitigation to cost as well. This factor was considerably reflected in the interview thematic analysis, supporting the need for further collaboration to utilise a broader set of skills and experience to dissect risks effectively.

\subsubsection{Group C2 - 4D BIM Timely Risk Reflection}

The second most important component factor for project diagnosis is identified to be the time in which 4D BIM can overturn the risks on projects, which came in at a total variance of $20.883 \%$ as presented in Table 4. It possesses similar factors to its preceding component factor of '4D BIM Thorough/Comprehensive Risk Reflection' in terms of its inclusiveness of numerous project factors and types but has more relation to the earlier nature of the risk mitigation; hence, the convention naming of '4D BIM Timely Risk Reflection'. With the factors being in relation to a proactive nature of identifying risks at an early stage, this suggests that the participants recognise early identification 
is key for achieving a better result. This includes the ability to influence the project design during the concept stage, as stated by Ciribini, et al. (2016) to deliver the project in a more efficient manner with better value for the end user. This signifies that while project teams want to address risks to the best of their ability, they also want to make sure this can be done in a timely manner that prevents holding up any other critical items the project may need again. This ultimately requires 4D BIM to be a process that again relates back to its planning efficiency factor and being a tool that is easy to use and produce an output in quick succession, regardless of the high amount of data/information it depends upon.

\subsubsection{Group C3 - 4D BIM Treatment and Relapse Prevention}

The last relevant component factor for project diagnosis is in relation to how 4D BIM allows the project team and the software itself devise solutions. This aligns with the Mordue \& Finch (2015) statement regarding the algorithmic approach it possesses to mitigate the identified risks and build further immunity for these risks becoming an issue again later down the line. The factor has a total variance of $17.715 \%$ as shown in Table 4. The name '4D BIM Treatment and Relapse Prevention' was devised with regards to the factors combining issues around subsequent processes beyond the identification of the risk and having a direct impact on moving the project forward. This suggests that the industry is possibly more open to the idea of having a more automated or standardised project/risk management procedure in place to aid the project and overcome issues. Nonetheless, such an automated system would still depend on the project team remaining in control of the process leading up to these offered solutions and incorporate any required amendments when enforcing the mitigation solution.

\section{Conclusion}

Construction projects are suffering regarding on-time delivery because of substandard project performance, which 4D BIM was identified as an appropriate solution to combat. To evaluate the effectiveness of the 4D BIM, this study was designed to investigate its impacts on project reliability, monitoring and diagnostics using an exploratory sequential mixed method. 
The key findings for 4D BIM impact on project reliability are that it enables the planning process to become a much more efficient process, allowing the planner to conduct typical responsibilities in a manner that derives more output. The second impact the 4D BIM has upon project reliability is the collaboration and education it can provide, bringing teams together for input across all the necessary members and provide a clear presentation to understand both the 4D BIM process and the genuine project plan. The final area identified for impacting on project reliability is the enhanced progress continuity/consistency, requiring measures in place to ensure the process does not falter. With project monitoring, 4D BIM could enhance the process with its additional assessment and directive capability, enhancing the planned progress vs actual progress to take more informed actions. The last impact of 4D BIM on project monitoring is the ability to incorporate technological appliances in conjunction with the 4D BIM process, thereby being able to accurately process progress data on site in conjunction with logic and expertise. The 4D BIM is identified to facilitate project diagnosis through its comprehensive risk reflection, being versatile enough to combat numerous issue types with the assistance of most project members upfront. Another area through which the 4D BIM can impact project diagnosis is with timely risk reflection, having the ability to identify well ahead of time where risks may occur to gain more leading on finding a solution. This links into the last impact factor with regards to treatment and relapse prevention, as the 4D BIM is also able to highlight solutions much more effectively than traditional planning means.

On full reflection of the purpose of 4D BIM and the contribution it can offer regarding project performance, it is still held in very high regard when evaluated amongst a wider sample of the industry. With 4D BIM being greatly seen as an efficient way to carry out the planning responsibility, the reasoning behind this is down to the fact that it is perceived mainly to be a tool championed by the planner, but it is actually a tool that would benefit massively from having other roles have direct input as well. Collaboration is commended in the sense that it can bring all parties into the mix to review the simulated plan, this is mainly down to it being a more interesting and immersive perspective for the planning process. This means that more roles need to have direct involvement, learn how to operate or at least contribute towards the 4D BIM planning process. With the added realisation that 4D BIM can implement better continuity of project progress with benefits that are evidently being experienced in a practical setting, this is can be attributed to the higher amount of effort that is required to produce the 4D BIM initially, which in turn equates to better input, better output. 
As far as the project monitoring with 4D BIM is concerned, it is a much more suitable platform for assessing the project progress, similarly to the point of progress continuity. This is down to its ability to accommodate for substantial progress data and been able to generate a realistic status report on the job, which could do with site engineers and managers having more responsibility to carry this out. This point was linked with the directive aspect that 4D BIM could generate a highly informing status reports, enabling the project team to take the advantage of the opportunities highlighted, which requires the involvement of more project members. 4D BIM can enhance the use of technological appliances to keep track of the progress, which means an automated approach is appealing to the industry for carrying out responsibilities while human validation is still required.

For project diagnosis, a comprehensive risk reflection is deemed a great impact, and this is down to the accommodating nature of the process, being applicable to represent vast scenarios and cater for the numerous roles to scope out as many issues as possible. This, again, depends heavily on the contributions of a diverse and collaborative team. Supplementary to being able to delve in deep to identify risks, the time in which they are identified is also a major impact 4D BIM can provide. This recognises that a system being fundamentally able to deeply analyse a risk could not be helpful if there is no time available afterwards to remedy the identified risks. The final way the 4D BIM impacts on project diagnosis is through its ability to get the team devising appropriate solutions with lasting effect, implying that automation should certainly be explored with 4D BIM along with human interpretation.

4D BIM brings out the best in people's efforts to produce great positive contributions towards project performance, as it thrives on great detail and user understanding to reflect these. The issue that lies with 4D BIM is the need for the process to become more streamlined to gain these benefits in a more manageable manner. Lack of familiarity and understanding of 4D BIM is also another main issue that is preventing the process impacting on project performance as effectively as it can. It is, therefore, important that companies provide more training regarding the process rather than just the use of the tool. This study also unearths a need for a more shared responsibility contributing to the 4D BIM process. The planner can get more out of 4D BIM in terms of output but does not really reduce any of their workloads. Getting the designer to build better quality data-rich models, the estimators and quantity surveyors allocating suitable budgets to the process, project managers enforcing measures to maintain the utilisation of 4D BIM is very essential to benefit from the 4D BIM. Site managers and engineers can also contribute by inputting the progress data of all activities, 
preferably with the assistance of more accurate methods of measurement technology. For it to maximize its full benefits, it is also important the process becomes more user-friendly while also incorporating a learning mechanism that would benefit from information of both failure and success. Further research could be necessary with regards to how automated the management of a project through BIM can become before the need for human input/logic becomes obsolete.

\section{References}

Braun, V. and Clarke, V., 2006. Using thematic analysis in psychology. Qualitative research in psychology, 3(2), pp.77-101.

Cabinet Office, 2011. Government Construction Strategy. London: Cabinet Office.

Ciribini, A. L. C., S. Mastrolembo Ventura, and Michela Paneroni. "Implementation of an interoperable process to optimise design and construction phases of a residential building: A BIM Pilot Project." Automation in Construction 71 (2016): 62-73.

Creswell, J. W. \& Creswell, D. J., 2018. Research Design: qualitative, quantitative \& mixed methods approaches. 5th ed. Los Angeles: SAGE Publications, Inc.

Eastman, C., Teicholz, P., Sacks, R. \& Liston, K., 2011. BIM Handbook: A Guide to Building Information Modeling for Owners, Managers, Designers, Engineers, and Contractors. 2nd ed. New Jersey: John Wiley \& Sons.

Egan, J., 1998. Rethinking construction, construction task force report for department of the environment, transport and the regions. ed: HMSO, London.

Fellows, R.F. and Liu, A.M., 2015. Research methods for construction. John Wiley \& Sons.

Field, A., 2013. Discovering Statistics Using IBM SPSS Statistics. 3rd ed. London: SAGE.

George, D. \& Mallery, P., 2003. Using SPSS for Windows Step by Step: a Simple Guide and Reference. 4th ed. Boston: Allyn and Bacon.

Hardin, B. \& McCool, D., 2015. BIM and Construction Management: Proven tools, methods, and workflows. 2nd ed. Indiana: Wiley.

Higgins, P. \& Cousins, P. T., 2013. Using the CIC Protocol. 1st ed. Westminster: NEC.

HM Government, 2016. Government Construction Strategy 2016-20. London: Crown Copyrights

Kerzner, H., 2013. PROJECT MANAGEMENT: A Systems Approach to Planning, Scheduling, and Controlling. 11th ed. New York: John Wiley \& Sons, Inc.

Larson, E. W. \& Gray, C. F., 2011. PROJECT MANAGEMENT: THE MANAGERIAL PROCESS. 5th ed. New York: McGraw-Hill/Irwin. 
Latham, M., 1994. Constructing the team: Joint review of procurement and contractual arrangements in the UK construction industry. Department of the Environment, UK.

Lu, W., Fung, A., Peng, Y., Liang, C. and Rowlinson, S., 2014. Cost-benefit analysis of Building Information Modeling implementation in building projects through demystification of timeeffort distribution curves. Building and environment, 82, pp.317-327.

McPartland, R., 2017. What is BIM?. [Online]. Available through: https://www.thenbs.com [Accessed 26 April 2018].

Merriam, S. B. (1998). Qualitative research and case study applications in education. Revised and expanded from" case study research in education." San Francisco, CA: Jossey-Bass Publishers

Mordue, S. \& Finch, R., 2015. BIM for Construction Health and Safety. 1st ed. Newcastle upon Tyne: RIBA Publishing.

National Audit Office, 2003. PFI: Construction Performance - A report by the Comptroller and Auditor General. London: The Stationery Office

Nunnally, J. C. \& Bernstein, I. H., 1994. Psychometric Theory. 3rd ed. New York: McGraw-Hill, Inc.

Sacks, R., Koskela, L., Dave, B.A. and Owen, R., 2010. Interaction of lean and building information modeling in construction. Journal of construction engineering and management, 136(9), pp.968-980.

Tabachnick, B. G. \& Fidell, L. S., 2001. Using multivariate statistics. 5th ed. Bolton: Pearson.

Yockey, R. D., 2011. SPSS DEMYSTIFIED: A Step-by-Step Guide to Successful Data Analysis. 2nd ed. New Jersey: Routledge.

Zhang, X., Azhar, S., Nadeem, A. and Khalfan, M., 2018. Using Building Information Modelling to achieve Lean principles by improving efficiency of work teams. International Journal of Construction Management, 18(4), pp.293-300.

Zhao, Q., Ma, Z., Hei, X., Zhu, Y. and Niu, J., 2017, December. A 3-D Structural Components Automatic Modeling Method Based on BIM. In Computational Intelligence and Security (CIS), 2017 13th International Conference on (pp. 59-63). IEEE. 